\title{
Elastic torsion effects in magnetic nanoparticle diblock-copolymer structures
}

\author{
L Schulz ${ }^{1,2}$, W Schirmacher ${ }^{1,3}$, A Omran ${ }^{1}$, V R Shah ${ }^{4}, \mathbf{P}$ Böni ${ }^{5}$, \\ W Petry ${ }^{1}$ and P Müller-Buschbaum ${ }^{1}$ \\ ${ }^{1}$ Physik-Department E13, Technische Universität München, James-Franck-Strasse 1, \\ D-85747 Garching, Germany \\ ${ }^{2}$ Dépt de Physique, Université Fribourg, Ch. du Musée 3, CH-1700 Fribourg, Switzerland \\ ${ }^{3}$ Institut für Physik, Universität Mainz, Staudinger Weg 7, 55099 Mainz, Germany \\ ${ }^{4}$ Nebraska Center for Materials and Nanoscience, 168 Behlen Lab, University of Nebraska \\ Lincoln, NE 68588-0111, USA \\ ${ }^{5}$ Physik-Department, E21, Technische Universität München, D-85747 Garching, Germany \\ E-mail: peter.mueller-buschbaum@ph.tum.de
}

\begin{abstract}
Magnetic properties of thin composite films, consisting of non-interacting polystyrene-coated $\gamma-\mathrm{Fe}_{2} \mathrm{O}_{3}$ (maghemite) nanoparticles embedded into polystyrene-block-polyisoprene P(S- $b$-I) diblock-copolymer films are investigated. Different particle concentrations, ranging from 0.7 to $43 \mathrm{wt} \%$, have been used. The magnetization measured as a function of external field and temperature shows typical features of anisotropic superparamagnets including a hysteresis at low temperatures and blocking phenomena. However, the data cannot be reconciled with the unmodified Stoner-Wohlfarth-Néel theory. Applying an appropriate generalization we find evidence for either an elastic torque being exerted on the nanoparticles by the field or a broad distribution of anisotropy constants.
\end{abstract}

\section{Introduction}

The magnetic properties of nanoparticle systems are of great interest because, firstly, they form model systems for understanding the mechanism of disordered magnetism [1] and, secondly, they have wide technological applications ranging from data storage [2], including high-frequency lossfree switching [3], to medical applications [4] and the creation of new materials $[5,6]$.

Since the time of the seminal theoretical papers of Stoner, Wohlfarth, Néel and Brown [1, 7, 8] and the experimental investigations of Bean and Livingston [9] a wealth of work has been published focusing on different aspects of the investigated heterogeneous magnetic materials [10].

The starting point of the interpretation of the magnetic properties of nanoparticles $[9,11,12]$ is the fact that below a certain particle volume only a single domain can exist, and the magnetic moment of the particle acts like a huge single spin, which can be treated classically ('superparamagnetism'). In the presence of magnetocrystalline anisotropy and/or shape anisotropy the 'superspins' become blocked below a certain blocking temperature $T_{\mathrm{B}}$, giving rise to divergent field-cooled (FC) and zero-field-cooled (ZFC) magnetization curves, a hysteresis loop and magnetic viscosity (slow logarithmic time dependence of the remnant magnetization). Such properties can be readily discussed and explained within the StonerWohlfarth-Néel approach [11, 12], although interpretations in terms of spin-glass models have been proposed invoking either interactions between the superspins [13] or postulating a surface spin-glass within each particle [14]. The latter interpretation had been put forward, because it has been known for a long time [15] that the saturation magnetization of ensembles of magnetic nanoparticles is much lower than the corresponding value of the bulk material. From this it must be concluded that, in fact, a nonmagnetic surface layer exists [16].

In this study magnetic properties of thin nanocomposite films, consisting of polystyrene (PS) coated $\gamma-\mathrm{Fe}_{2} \mathrm{O}_{3}$ (maghemite) nanoparticles embedded into disordered polystyrene-block-polyisoprene ( $\mathrm{P}(\mathrm{S}-b-\mathrm{I}))$ diblock-copolymer matrices, are investigated. The diameter of the nanoparticles 


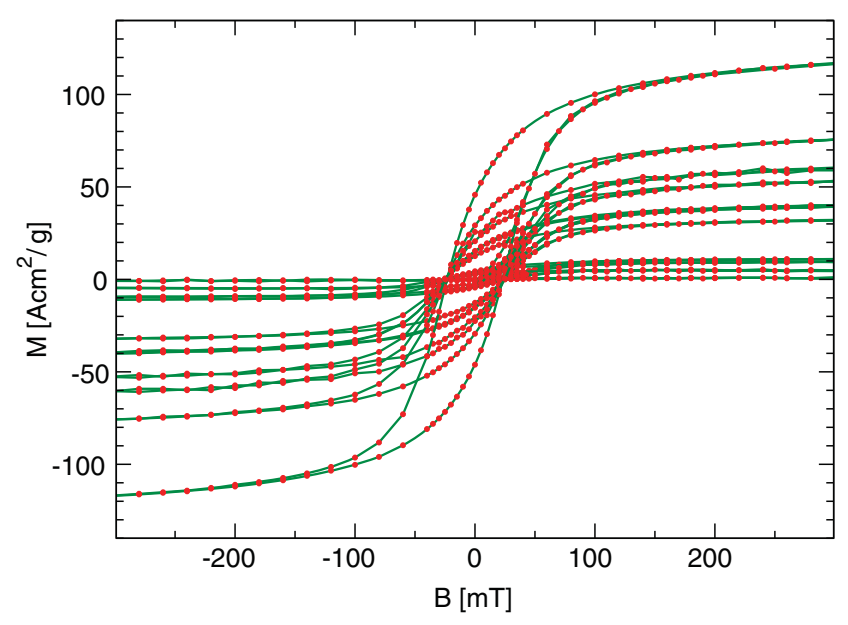

Figure 1. Magnetization curves $M$ versus $B$ at $T=2 \mathrm{~K}$ for the following weight percentages (from low to high $M$ ) $0.7,2,4,8,14$, $16,18,20,25,43 . B$ is the external field $B=H_{\text {ext }} / \mu_{0}$.

is chosen to be only slightly smaller than the characteristic spacing of the micro-phase separation structure of the diblockcopolymer matrix. This results in a heterogeneous nature of the diblock-copolymer matrix, forcing the PS-coated particles to reside only inside the PS regions. Thus, we achieve an optimal spatial separation of the particles from one another. The disordered matrix ensures the absence of correlation effects, which might arise from an ordered micro-phase separation structure.

We are able to demonstrate from our measured magnetization data that inter-particle interaction effects can be ruled out. This leads to the possibility of a realistic comparison with the classical superparamagnetic theory. Our data show superparamagnetism at high temperatures and blocking phenomena at low ones which is to be expected for a material with magnetocrystalline anisotropy. The phenomena can be accounted for reasonably well within the StonerWohlfarth-Néel model. However, the hysteresis curves differ appreciably from what one expects from Stoner-Wohlfarth theory [12]. We shall demonstrate two alternative models that can possibly explain the hysteresis data, either by assuming a mechanical torsion of the particle produced by the applied field or a spread in the anisotropy of the nanoparticles.

\section{Samples and measurements}

Our samples belong to a special class of nanocomposites: polymer-metal oxides. Such materials were investigated optically, electrically [17], mechanically [18] and structurally [19]. Lauter-Pasyuk et al examined the structure of thin lamellae forming diblock-copolymer films with incorporated polymer-coated nanoparticles. Due to polystyrene coating of the nanoparticles, a well controlled spatial distribution of the nanoparticles within the film was achieved [20, 21]. Here we focus on thick diblock-copolymer films with a heterogeneous inner morphology. Because the volume fraction of polyisoprene (PI) in the investigated copolymer is $f_{\mathrm{PI}}=N_{\mathrm{PI}} / N=0.44$, lamellar structures form without

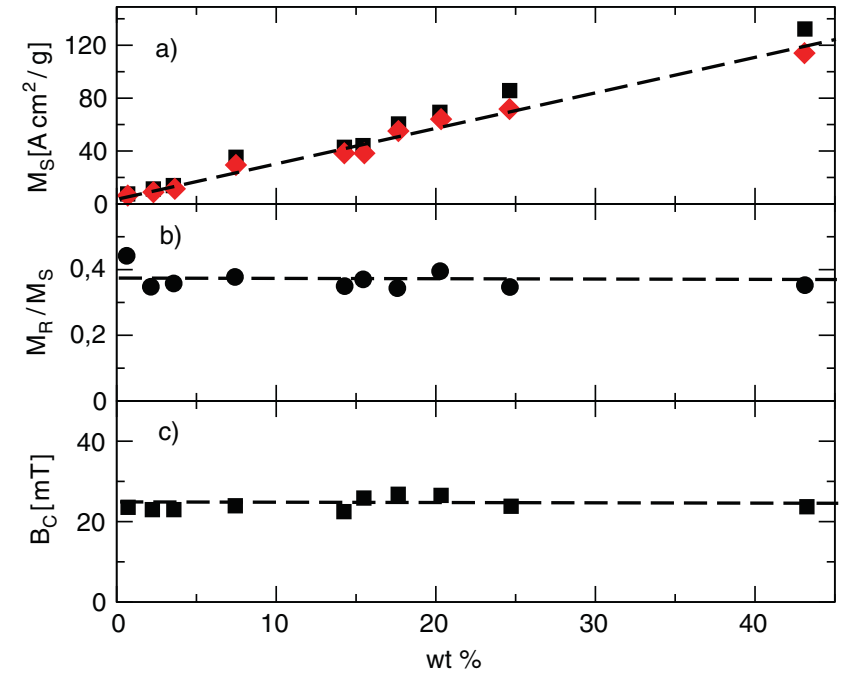

Figure 2. (a) Saturation magnetization at 2 and $300 \mathrm{~K}$ versus concentration. (b) Relative remnant magnetization at $2 \mathrm{~K}$ versus concentration. (c) Coercive field $B_{\mathrm{C}}$ at $2 \mathrm{~K}$ versus concentration.

the addition of nanoparticles due to micro-phase separation [22, 23]. In this investigation, the molar mass of the used diblock-copolymer is $24500 \mathrm{~g} \mathrm{~mol}^{-1}$, which gives rise to a lamellar spacing of $13 \mathrm{~nm}$. Maghemite $\left(\gamma \mathrm{Fe}_{2} \mathrm{O}_{3}\right)$ nanoparticles covered with polystyrene hairs were synthesized from $\alpha$-lithium polystyrene sulfonated (LPSS) in toluene solution by the addition of an aqueous mixture of $\mathrm{FeCl}_{2}$ and $\mathrm{FeCl}_{3}$. The average nanoparticle diameter is $d_{0}=$ $10 \mathrm{~nm}$ as determined with scattering experiments. Since the nanoparticles are coated with polystyrene, they show an affinity to the polystyrene blocks [20, 24]. Samples with nanoparticle weight concentrations of $c=0,0.7,2,4$, $8,14,16,18,20,25$ and 43 wt $\%$ were prepared by the solution casting technique to allow for an equilibration of the morphology. Precleaned silicon wafers were used as substrates.

The magnetic properties of our samples were investigated with a physical property measurement system (PPMS) from Quantum Design. Figure 1 shows our measured magnetization curves at $T=2 \mathrm{~K}$ for all concentrations. In figure 2 we have plotted the saturation magnetization $M_{\mathrm{S}}$, the relative remanence $M_{\mathrm{R}} / M_{\mathrm{S}}$ and the coercivity field $B_{\mathrm{C}}$ versus concentration. From the linear increase of $M_{\mathrm{S}}$ with concentration and the concentration independence of $M_{\mathrm{R}} / M_{\mathrm{S}}$ and $B_{\mathrm{C}}$ we conclude that the particles do not interact. This is obviously due to the fact that the single PS-coated particles are located in PS pockets of the polymer matrix. In particular we have no evidence for cluster formation.

In figure 3 the magnetic moment curves at $T=2$ and $300 \mathrm{~K}$ for two samples are compared. We have also measured the AC susceptibility of the samples at a frequency of $f=1 \mathrm{kHz}$. The data show the same linear concentration dependence as the DC magnetization curves. We also measured the $\mathrm{AC}$ and $\mathrm{DC}$ quantities with a FC and ZFC protocol. Below $T \approx 100 \mathrm{~K}$ the $\mathrm{FC}$ and $\mathrm{ZFC}$ magnetizations differ from each other. In our opinion this feature is not 


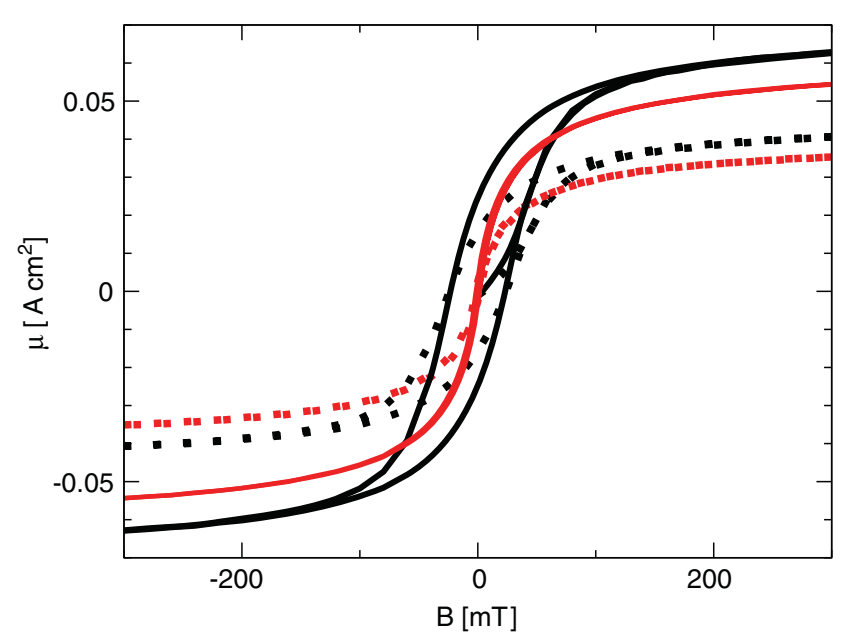

Figure 3. Magnetic moment curves $\mu$ versus $B$ for two different nanoparticle concentrations $c=25 \mathrm{wt} \%$ (dotted curves) and $c=42 \mathrm{wt} \%$ (continuous curves) at $T=300 \mathrm{~K}$ (no hysteresis) and $T=2 \mathrm{~K}$ (with hysteresis)

caused by a spin-glass behavior [5, 14], but reflects the fact that particles with volumes larger than

$$
V_{\mathrm{B}}(T)=\left(k_{\mathrm{B}} T / K\right) \ln \left(t_{\mathrm{exp}} v_{0}\right)
$$

are blocked. $K$ is the anisotropy barrier per volume, $t_{\exp }$ the experimental timescale and $v_{0}$ the attempt frequency for transitions across the barrier. From the temperature dependence of the out-of-phase AC susceptibility $\chi^{\prime \prime}(f, T)$ we can extract the volume distribution of the nanoparticles $P(V)$ because it can be shown [11] that for a single particle $\chi^{\prime \prime}(f, T) \propto \delta\left(V-V_{f}(T)\right)$, with

$$
V_{f}(T)=\left(k_{\mathrm{B}} T / K\right) \ln \left(v_{0} / 2 \pi f\right) .
$$

We see from figure 4 that the data can be fitted well with a log-normal distribution

$$
P(V)=P_{0} \frac{1}{V} \mathrm{e}^{-\frac{1}{2 \delta^{2}}\left(\ln \left(V / V_{0}\right)\right)^{2}}
$$

with width parameter $\delta=0.8 . P_{0}=1 / \delta \sqrt{2 \pi}$ (normalization) and $V_{0}=(\pi / 6) d_{0}^{3}$.

The fact that the function $\chi^{\prime \prime}(T)$ can be reconciled with the expected log-normal volume distribution of isolated nanoparticles corroborates our conclusion that the particles do not interact. If the particles form magnetic clusters one would expect a high-temperature deviation from the single-particle distribution.

\section{Data analysis}

\subsection{The Stoner-Wohlfarth model}

We turn now to a detailed discussion of the low-temperature hysteresis based on the following model with uni-axial anisotropy $[1,11,12,25]$ :

$$
\mathcal{H}_{0}=2 K V\left(\frac{1}{2} \sin ^{2}(\theta)-h \cos (\alpha-\theta)\right)
$$

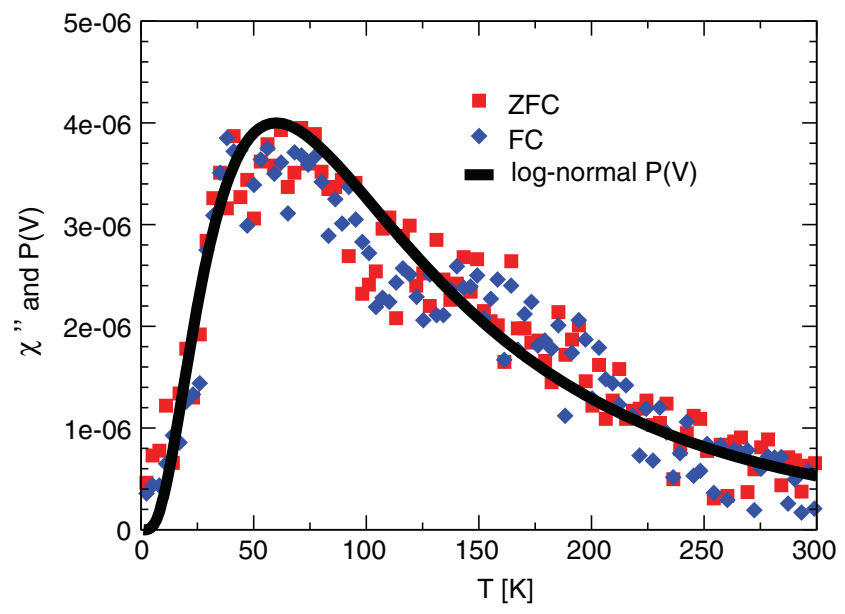

Figure 4. Out-of-phase susceptibility at $\omega / 2 \pi=1 \mathrm{kHz}$ compared with a $\log$-normal function with width parameter $\delta=0.8$.

$\theta$ is the angle between the magnetization and the easy axis and $\alpha$ the angle between the field $B$ and the easy axis. $h=$ $B M_{\mathrm{S}} / 2 \mathrm{~K}$ is the dimensionless field parameter, where $M_{\mathrm{S}}$ is the saturation magnetization. Minimizing (4) with respect to $\theta$ one obtains an analytic expression for $h$

$$
\begin{gathered}
h(\theta, \alpha)=\left(\cos ^{2}(\alpha-\theta)-\frac{1}{2}\right) \frac{\sin (2 \alpha)}{\sin (\alpha-\theta)} \\
-\cos (\alpha-\theta) \cos (2 \alpha) .
\end{gathered}
$$

Out of this one gets the dimensionless magnetization of the particle ensemble as

$$
m_{0}(h)=\langle\cos (\alpha-\theta(h))\rangle_{\alpha}
$$

where $\langle\cdots\rangle_{\alpha}$ denotes an average over $\alpha$ ranging from 0 to $\pi / 2$, and $\cos (\alpha-\theta(h))$ is the component of magnetization along the external field. The magnetization of the sample is then $M_{0}=m_{0} M_{\mathrm{S}}=\mu / V_{\mathrm{S}}$, where $\mu$ is the magnetic moment and $V_{\mathrm{S}}$ is the volume of the nanocomposite sample.

The hysteresis curve has to be calculated using the switching condition

$$
h=h_{\mathrm{sw}}^{0}=-\left[\sin ^{2 / 3}(\alpha)+\cos ^{2 / 3}(\alpha)\right]^{-3 / 2}
$$

as described in the literature [12, 25]. Interestingly enough the particle volume drops out of these low-temperature calculations. At finite temperatures, though, the switching condition is modified as follows:

$$
h_{\mathrm{sw}}(\alpha, T)=h_{\mathrm{sw}}^{0}\left[1-\left(\frac{V_{\mathrm{B}}(T)}{V}\right)^{\frac{2}{3}}\right] \text {. }
$$

The resulting magnetization $m_{0}(h)$ is the outermost curve in figure 5. Clearly it cannot be reconciled with the measured data at $2 \mathrm{~K}$.

\subsection{The elastic torque model}

We therefore include into our model the possibility of an elastic torsion of the nanoparticles due to the applied field. Assuming 


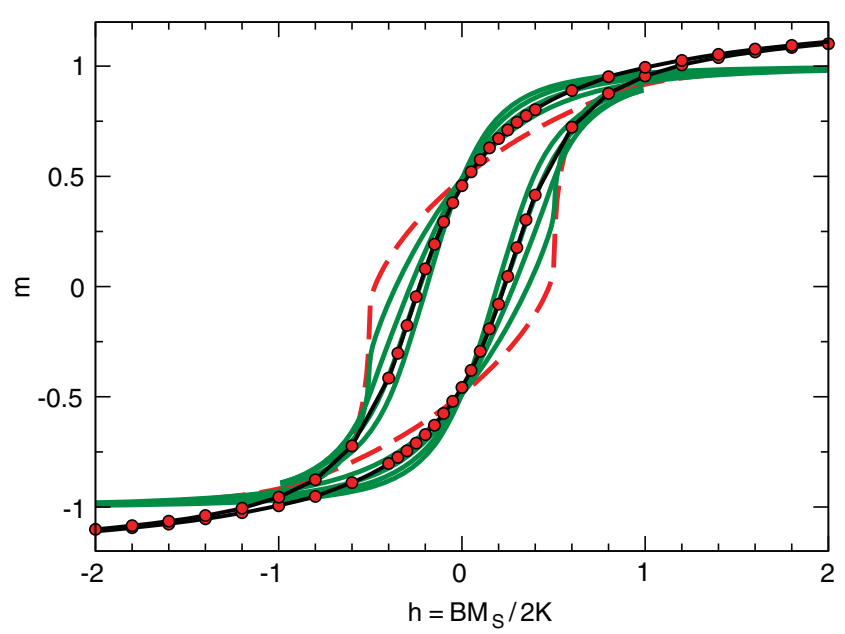

Figure 5. Calculated hysteresis curves for different elasticity parameters. Red dashes: $\gamma=0$ (Stoner-Wohlfarth theory), green lines (inwards) $\gamma=0.5,1.0,1.5,2.0$. Symbols: measured data of the $45 \%$ sample at $T=2 \mathrm{~K}$.

roughly spherical particles, the torque needed to displace a particle of volume $V$ by an angle $\Delta \alpha=\alpha-\alpha_{0}$ is given by [26]

$$
\tau=6 G V\left(\alpha-\alpha_{0}\right) .
$$

Here, $G$ is the shear modulus. As the external field exerts this torque, the torsion angle is given by

$$
\Delta \alpha=\alpha-\alpha_{0}=-\frac{1}{6 G V} M_{\mathrm{S}} V B \sin (\alpha-\theta) .
$$

In order to include this effect we add a corresponding term to the Hamiltonian

$$
\mathcal{H}=\mathcal{H}_{0}+2 K V\left(\frac{1}{2 \gamma}\left(\alpha-\alpha_{0}\right)^{2}\right)
$$

with elasticity parameter $\gamma=K / 3 G$. Minimizing (11) with respect to $\theta$ and $\alpha$ one obtains a closed set of equations for $\theta\left(h, \alpha_{0}\right)$ and $\alpha\left(h, \alpha_{0}\right)$ from which the magnetization follows via (6), where the average is now over $\alpha_{0}$. Figure 5 shows the resulting hysteresis curves with $\gamma$ between 0 and 2 . We take the good agreement with the data for $\gamma \approx 1$ as evidence for the presence of the elastic mechanism. As the bulk value for the magnetocrystalline anisotropy of maghemite is $K=$ $4.7 \times 10^{3} \mathrm{~J} \mathrm{~m}^{-3}$ we see that we are dealing with values of a shear modulus $G$ of the order of $\mathrm{kPa}$. This is an extremely low value in comparison with typical shear moduli of macroscopic elastic materials. However, as the nanoparticles are loosely packed inside the PS pockets, and because the shear stiffness of our films is at the borderline of yielding, we consider such a value to be realistic.

\subsection{Temperature dependence}

We now consider the temperature dependence of the magnetization. At intermediate temperatures $T$ particles with a volume larger than the blocking volume $V_{\mathrm{B}}(T)$ are blocked (see equation (1)). We make the ansatz for decreasing $h>0$ (upper hysteresis curve)

$$
m(T, B)=\int_{0}^{V_{\mathrm{B}}(T)} \mathrm{d} V P(V)\left\langle m_{\mathrm{eq}}\left(\alpha_{0}, V\right)\right\rangle_{\alpha_{0}}+m_{0} c_{\mathrm{B}}(T) .
$$

The first term is the contribution of the non-blocked particles, which we assume to be in thermal equilibrium and not affected by the elastic mechanism. $m_{\text {eq }}$ is given by [11]

$$
m_{\mathrm{eq}}(V, B, T)=M_{\mathrm{S}} V \frac{\partial \ln \mathcal{Z}}{\partial \xi}
$$

where $\mathcal{Z}$ is the partition function

$$
\mathcal{Z}=\int_{0}^{\pi} \mathrm{d} \theta \sin (\theta) \mathrm{e}^{\sigma \cos (\theta)^{2}} I_{0}[\xi \sin (\theta) \sin (\alpha)] \mathrm{e}^{\xi \cos (\theta) \cos (\alpha)}
$$

with $\xi=M_{\mathrm{S}} B V / k_{\mathrm{B}} T . I_{0}(x)$ is the modified Bessel function of order zero.

The second contribution is that of the blocked superspins. $m_{0}$ is the angle-averaged magnetization at $T=0$ (including the elastic torsion effect), and $c_{\mathrm{B}}(T)=\int_{V_{\mathrm{B}}}^{\infty} \mathrm{d} V P(V)$ is the concentration of the blocked particles.

In order to obtain the switching behavior for decreasing $h<0$, we use the approximation of [12] for the barrier lowering by a reversed field

$$
\Delta E=K V\left[1-h / h_{\mathrm{sw}}^{0}\right]^{1.5} .
$$

This enables particles with volumes between $V_{\mathrm{B}}$ and $V_{\mathrm{B}}=$ $V_{\mathrm{B}} /\left[1-h / h_{\mathrm{sw}}^{0}\right]^{1.5}$ to switch, and we obtain for the switching behavior of the blocked particles

$$
\begin{aligned}
& m_{\text {blocked }}(h)=\left(m_{0,1}-m_{0,2}\right) \Theta\left(h-h_{\mathrm{sw}}\right) \int_{V_{\mathrm{B}}}^{\infty} \mathrm{d} V P(V) \\
& \quad+m_{0,2} c_{\mathrm{B}}(T) .
\end{aligned}
$$

Here $m_{0, i}$ are the two branches of the zero-temperature hysteresis curves (including the elastic effect), averaged over $\alpha_{0}$ and $\Theta(x)$ is the Heaviside step function. In figures 6 and 7 we compare the calculated finite-temperature magnetization curves with our measured ones between 2 and $100 \mathrm{~K}$. We see that we obtain rather good agreement. A striking feature is that the slope near $B=0$, which from a naive point of view should obey a Curie law, becomes temperature independent as a combined result of the polydispersivity and the elastic effect.

Figure 6 also shows a comparison of the measured remanence and the calculated one as a function of temperature obtained by evaluating the magnetization of blocked particles at $h=0$ :

$$
\begin{aligned}
& m_{r}(T)=\left.\langle\cos \phi\rangle_{\alpha}\right|_{h=0} \int_{V_{\mathrm{B}}(T)}^{\infty} \mathrm{d} V P(V) \\
& =\frac{1}{4} \operatorname{erfc}\left(\frac{1}{\delta \sqrt{2}} \ln \frac{V_{\mathrm{B}}(T)}{V_{0}}\right) .
\end{aligned}
$$

The theoretical coercivity values could only be extracted from numerical calculations, but agree well with the experimental data (figure 7). 


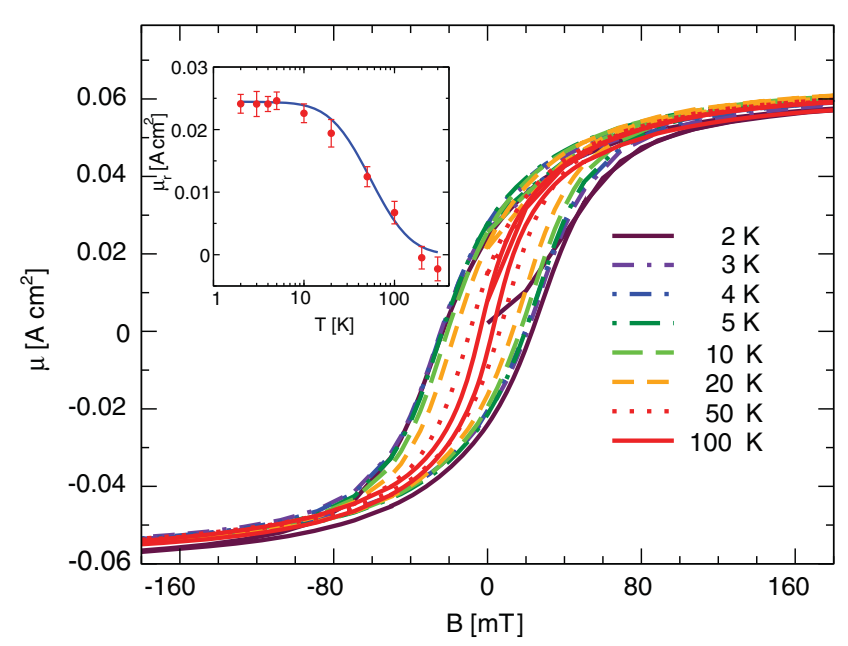

Figure 6. Measured hysteresis curves at several temperatures. Inset: measured remanence (symbols) versus theory (line).

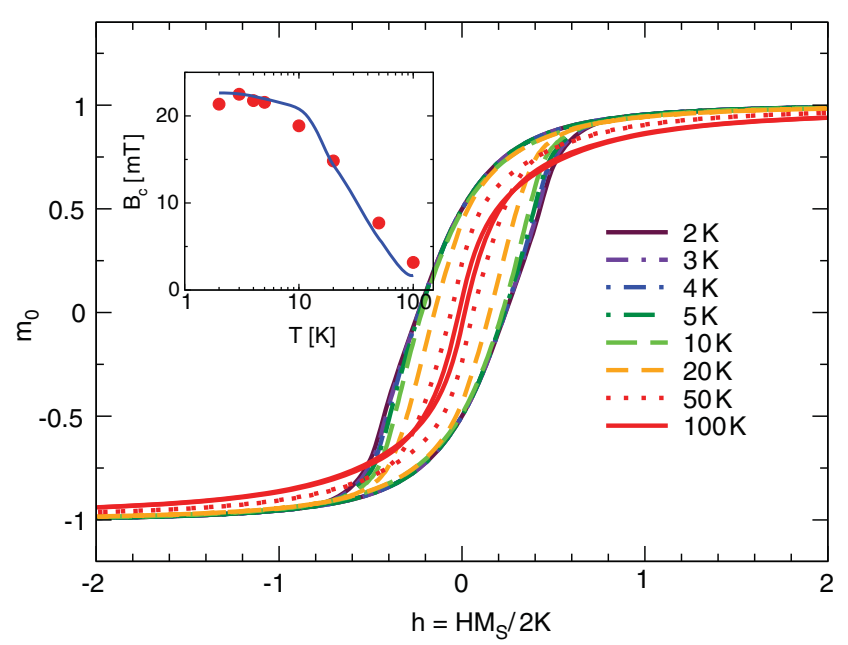

Figure 7. Calculated hysteresis curves at several temperatures. Inset: measured coercivity (symbols) versus theory (line).

\section{Discussion and alternative model}

In light of our investigations it is worthwhile to discuss the role of the surface layer of the nanoparticles. The saturation magnetization of figure 2 extrapolates to the value of $\approx 30 \mathrm{~A} \mathrm{~m}^{2} \mathrm{~kg}^{-1}$, a value, which is much lower than the maghemite bulk value of $76 \mathrm{~A} \mathrm{~m}^{2} \mathrm{~kg}^{-1}{ }^{6}$ Because the magnetic properties of our samples can be fully understood in terms of the superparamagnetic and blocking model, we assume that this surface layer is just magnetically inert.

An alternative approach to explaining the low-temperature magnetization behavior is based on including a spread in the anisotropy constant $K$ that could possibly arise from shape and surface effects. We make an ansatz with a normal distribution

$$
P(\kappa)=\frac{1}{\sqrt{2 \pi} \delta_{K}} \exp \left(-\frac{\left(K-K_{0}\right)^{2}}{2 \delta_{K}^{2}}\right)
$$

6 The saturation magnetization value of $\approx 30 \mathrm{~A} \mathrm{~m}^{2} \mathrm{~kg}^{-1}$ nicely agrees to the one found by Millan et al [16] for particles of $10 \mathrm{~nm}$ diameter.

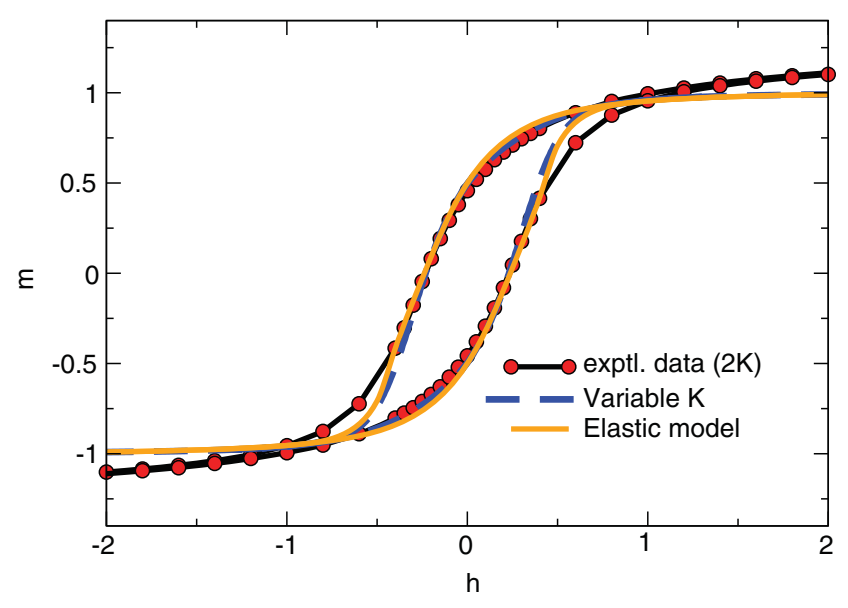

Figure 8. Low-temperature curves for anisotropy spread compared to elastic torque model and experimental data at $2 \mathrm{~K}$.

where $K_{0}$ is the average anisotropy constant, and $\delta_{K}$ is the distribution width. We find that the low-temperature magnetization curve is reproduced best with $\delta_{K}=0.4 K_{0}$ (figure 8) and the resulting curve compares well to the one obtained in the elastic torque model. The finite-temperature behavior might be explained within this model as well. Here a modification of the switching condition of blocked particles for every value of $K$ must be taken into account. In addition, the possibility also remains that both models are simultaneously valid, where a smaller spread in $K$ and smaller value of $\gamma$, i.e. higher shear stiffness, would also reproduce the same results.

\section{Conclusion}

In conclusion, we produced a nanocomposite material with well-separated magnetic nanoparticles embedded in a block copolymer matrix. The superparamagnetic model of Stoner, Wohlfarth, Néel and Brown [1, 7, 8, 11, 12], or generalized versions of it as presented above, therefore can be applied. We find that the magnetic properties of our composite materials can only be explained, if in addition to the thermodynamics of the superspins either an elastic torsion mechanism or a statistical distribution of the anisotropy constants $K$ is taken into account. The elastic torsion effect-as well as the possible $K$ distribution considerably deforms the hysteresis curves and consequently the coercivity. Further exploration of the elastic torsion effect may open the possibility to tailor the magnetic properties of copolymer-nanoparticle composites and might be an important step towards micro- and nanomagnetic applications.

\section{Acknowledgments}

We are grateful to Bernd Rellinghaus, Manuel Richter, Ulrich Rössler and Rolf Schilling for helpful discussions. Support by Barbara Russ in performing the measurements is gratefully acknowledged. We are grateful to Andreas Michels for critically reading the manuscript. 


\section{References}

[1] Stoner E C and Wohlfarth E P 1948 Phil. Trans. R. Soc. A 240500

Stoner E C and Wohlfarth E P 1991 IEEE Trans. Magn. 273475 (reprinted)

[2] Thompson D A and Best J S 2000 IBM J. Res. Dev. 44311

[3] Sukhov A and Berakdar J 2009 Phys. Rev. Lett. 102057204

[4] Pankhurst Q A et al 2003 J. Phys. D: Appl. Phys. 36 R167

[5] Dormann J and Spinu L 1998 J. Magn. Mater. 187 L193

[6] Sun Y et al 2003 Phys. Rev. Lett. 91167206

[7] Néel L 1949 Ann. Geophys. (CNRS) 599

[8] Brown W F 1963 Phys. Rev. 1301677

[9] Bean C P and Livingston J D 1959 J. Appl. Phys. 30 S120

[10] Fiorani D (ed) 2005 Surface Effects in Magnetic Nanoparticles (Berlin: Springer)

[11] García-Palacios J L 2000 Adv. Chem. Phys. 1121

[12] Wernsdorfer W 2001 Adv. Chem. Phys. 11899
[13] Jönsson P, Jonsson T, García-Palacios J and Svendlindth P 2000 J. Magn. Magn. Mater. 222219

[14] Martinez B et al 1998 Phys. Rev. Lett. 80181

[15] Berkowitz A E et al 1973 Phys. Rev. Lett. 34594

[16] Millan A et al 2007 J. Magn. Magn. Mater. 312 L5

[17] Schürmann U et al 2006 Thin Solid Films 515801

[18] Jeon J et al 2006 J. Power Sources 1621304

[19] Park M J et al 2006 Langmuir 221375

[20] Lauter-Pasyuk V et al 2003 Langmuir 197783

[21] Lauter-Pasyuk V et al 2004 Physica B 350 E939

[22] Bates F and Fredrickson G 1990 Ann. Rev. Phys. Chem. 41525

[23] Khandpur A et al 1995 Macromolecules 288796

[24] Abul Kashem M M et al 2007 Macromolecules 405075 Abul Kashem M M et al 2007 Macromolecules 412186

[25] Getzlaff M 2008 Fundamentals of Magnetism (Heidelberg: Springer)

[26] Wilhelm C et al 2003 Phys. Rev. E 67011504 